\title{
Fixed-ratio performance and subsequent extinction in rats prenatally exposed to ethanol
}

\author{
EDWARD P. RILEY, NEIL R. SHAPIRO, ELIZABETH A. LOCHRY, \\ and JOHN P. BROIDA \\ State University of New York, Albany, New York 12222
}

\begin{abstract}
Pregnant Long-Evan rats were fed isocaloric liquid diets containing $35 \%, 23 \%, 11 \%$, or $0 \%$ ethanol-derived calories (EDC) from Day 5 to Day 20 of gestation. Shortly after weaning, male offspring of these mothers were tested for the acquisition and extinction of a fixed-ratio (FR) barpress response. As the schedule requirement was progressively increased from FR 2 to FR 33, the 35\% (EDC) offspring consistently had the lowest response rates, $23 \%$ and $11 \%$ (EDC) offspring were intermediate, and the $0 \%$ (EDC) progeny had the highest response rates $(p<.01)$. During extinction, this pattern was reversed; the number of responses increased as a function of prenatal alcohol exposure $(p<.025)$.
\end{abstract}

Alcohol has recently been recognized as a functional teratogen, affecting subsequent development and behavior when administered prenatally. For example, in rodents, alcohol exposure in utero results in retarded intrauterine and postnatal growth (Henderson \& Schenker, 1977; Martin, Martin, Sigman, \& Radow, 1977; Riley, Lochry, Shapiro, \& Baldwin, 1979; Tze \& Lee, 1975) as well as delayed behavioral development (Demers \& Kirouac, 1978; Shaywitz, Klopper, \& Gordon, 1976). The most commonly reported behavioral consequence of prenatal alcohol exposure is hyperactivity as measured in an open field (Bond \& DiGiusto, 1976, 1977; Branchey $\&$ Friedhoff, 1976; Shaywitz et al., 1976) and running wheel (Martin, Martin, Sigman, \& Radow, 1978). However, hyperactive behavior is by no means the sole manifestation of prenatal alcohol exposure. Rodents subjected to alcohol exposure in utero have also been reported to exhibit poorer maze learning performance (Shaywitz et al., 1976) and deficits in shuttle avoidance (Bond \& DiGiusto, 1978; Shaywitz et al., 1976) and passive avoidance (Riley, Lochry, $\&$ Shapiro, 1979) when compared to controls. These animals have also been shown to require a greater number of trials prior to spontaneous alternation in a $\mathrm{T}$-maze and prior to learning the reversal of a discriminated shock-escape task in a similar apparatus (Riley et al., 1979b).

The present study was conducted to examine oper-

This work was partially supported by DHEW Research Grants AA03249-02 and -03 from the National Institute on Alcohol Abuse and Alcoholism and a grant from The National Council on Alcoholism to E. P. Riley. E. Lochry was an NCA predoctoral fellow during the course of this research. Reprint requests should be addressed to Edward P. Riley, Department of Psychology, SUNYAlbany, Albany, New York 12222. ant, fixed-ratio behavior in animals prenatally exposed to alcohol. In a previous study, Martin et al. (1977) found that rats, exposed to alcohol prenatally and while nursing, received fewer reinforcements on both CRF and FR-10 schedules than did controls. During the 2nd week of FR 10, the differences between the groups diminished. However, as the Martin et al. (1977) study was not designed to separate prenatal and postnatal effects of alcohol exposure, the present investigation was conducted to examine FR behavior in animals exposed solely during the prenatal period.

\section{METHOD}

\section{Subjects}

Parent animals were Long-Evans rats obtained from Blue Spruce Farms, Inc., Altamont, New York. Each evening, females were individually placed with a male overnight until a vaginal smear taken the following morning indicated Day 1 of pregnancy. These pregnant females were then weighed and housed in standard breeding cages. All animals were maintained on a 12-h light-dark cycle, with free access to food and water except as noted.

Thirty-two of these females were provided with liquid diets containing various amounts of the total calories in the form of ethanol from Day 5 through Day 21 of pregnancy. Ten females were given access to diets with $35 \%$ ethanol-derived calories (EDC), seven had 23.3\% EDC diets, eight were given $11.7 \%$ EDC diets, and seven were administered $0 \%$ EDC diets. A group of six females was maintained on lab chow and water throughout their pregnancies to control for liquid diet administration. All diets, which provided $1 \mathrm{kcal} / \mathrm{ml}$, were mixed and administered daily and served as the sole source of nutrition during the period of administration. The compositions of the diets are provided in Table 1.

A pair-feeding procedure was employed to equate caloric intake across the various groups. Animals assigned to the $35 \%$ group were allowed free access to their diets, while animals in the remaining liquid-diet groups were yoked to a $35 \%$ animal and received only the amount consumed by this $35 \%$ female on a $\mathrm{ml} / \mathrm{kg}$ weight basis. Maternal weights were reassessed at 5-day intervals during pregnancy to obtain more precise control over the pairfeeding routine. Blood alcohol concentrations were assessed on 
Table 1

Liquid Diet Compositions per $100 \mathrm{ml}^{*}$

\begin{tabular}{lrrrr}
\hline & $35 \%$ & $23.3 \%$ & $11.7 \%$ & \multicolumn{1}{c}{$0 \%$} \\
\hline Nutrament $\dagger$ & 64.10 & 64.10 & 64.10 & 64.10 \\
$95 \%$ EtOH $\dagger$ & 6.67 & 4.44 & 2.23 & .00 \\
Sucrose $\dagger \dagger$ & .00 & 2.93 & 5.83 & 8.75 \\
Vitamins $\dagger \dagger$ & .25 & .25 & .25 & .25 \\
\hline
\end{tabular}

*Water added for a total volume of $100 \mathrm{ml}$. fIn milliliters. t+In grams.

Days 10 and 15 of gestation, using a relatively nontraumatic procedure described by Lester (1963).

Newborn pups were weighed and body length was assessed at birth, when litters were culled to 10 pups. The offspring were again weighed and measured for linear growth at 10 and 20 days of age. Pups were weaned at 21 days of age and housed two per cage. At 25 days of age, male offspring were selected randomly from each of the five groups and placed on a 23-h deprivation schedule $(\mathrm{ns}=9,8,10,8$, and 10 for the $35 \%, 23 \%, 11 \%, 0 \%$ EDC, and lab chow groups, respectively). All litters within each prenatal treatment condition were represented by at least one animal, but in no instance were more than two animals from a litter selected. One subject in the $35 \%$ group was eliminated from the experiment for reasons unrelated to the experimental treatment.

\section{Apparatus}

Three standard operant conditioning chambers (Coulbourn Instruments, Inc.) were used. Each box had a single lever protruding from the right side of the front wall $1.5 \mathrm{~cm}$ into the box and $2.5 \mathrm{~cm}$ above the floor. A food cup, recessed and centered on the front wall $3.5 \mathrm{~cm}$ from the lever, was used to deliver $45-\mathrm{mg}$ Noyes pellets. Each chamber was illuminated by a $28-\mathrm{V}$ houselight and was enclosed in a sound-attenuating box equipped with a ventilating fan. Programming and data recording were accomplished by means of solid state logic located in an adjacent room.

\section{Procedure}

At approximately 30 days of age, each subject was shaped to barpress on a continuous reinforcement schedule (CRF). Subjects were given a total of three 20 -min training sessions with additional shaping given when required, followed by one 20 -min session of CRF with no experimenter intervention. Subsequently, the fixed-ratio (FR) requirement was increased every 3 days from FR 2 to FR 3, FR 5, FR 9, FR 17, and FR 33. After 3 days on the FR-33 schedule, the rats were given six extinction sessions which were also $20 \mathrm{~min}$ in duration.

\section{RESULTS}

The mean number of reinforcements received as a function of response requirement for offspring from each of the four liquid diet groups is presented in Figure 1. As indicated, there is a distinct tendency for the number of reinforcements to decrease as the level of prenatal alcohol increases. This pattern appears to be consistent across all of the schedules used. An analysis of variance on these data, with groups as a between-subjects factor and schedule and days as within-subject factors, as well as subsequent analyses of trend (Keppel, 1973), was conducted to determine the significance of these effects. An overall effect of the percentage of EDC supplied to the mother proved significant $[\mathrm{F}(3,30)=3.01, \mathrm{p}<.05]$, and decomposition into trend components indicated that only the linear trend was statistically significant $[\mathrm{F}(1,30)=8.23, \mathrm{p}<.01]$. Additionally, the interaction of days and schedule was significant $[F(10,300)=$ $3.91, \mathrm{p}<.01$ ], as were the main effects of days and schedule. However, neither of these variables interacted with the alcohol treatment conditions.

While prenatal alcohol exposure appeared to decrease leverpress performance during the fixed-ratio reinforcement sessions, alcohol-exposed offspring, with the exception of the $11 \%$ group, tended to respond more than $0 \%$ controls across the six extinction sessions $(\overline{\mathrm{X}} \mathrm{s}=125.73,129.60,102.25$, and 117.31 for the $35 \%, 23 \%, 11 \%$, and $0 \%$ groups, respectively). However, since standard deviations appeared to be functions of the cell means, raw scores were transformed by taking the $\log (x+1)$, as described in Winer $(1971$, p. 400$)$. The mean transformed number of leverpresses for each group are presented as a function of session in Figure 2. An analysis of variance performed on the transformed scores revealed a significant linear component of the diet group variable $[\mathrm{F}(1,30)=6.01, \mathrm{p}<.025]$. This linear function accounted for approximately $74 \%$ of the between-group variance, while the quadratic and cubic trend components failed to reach significance. Additionally, a significant session effect was evident $[F(5,150)=117.66, p<.001]$, reflecting the overall decline in responding across sessions $[\mathrm{F}(1,30)$ $=333.09, \mathrm{p}<.001]$. This decline occurred similarly

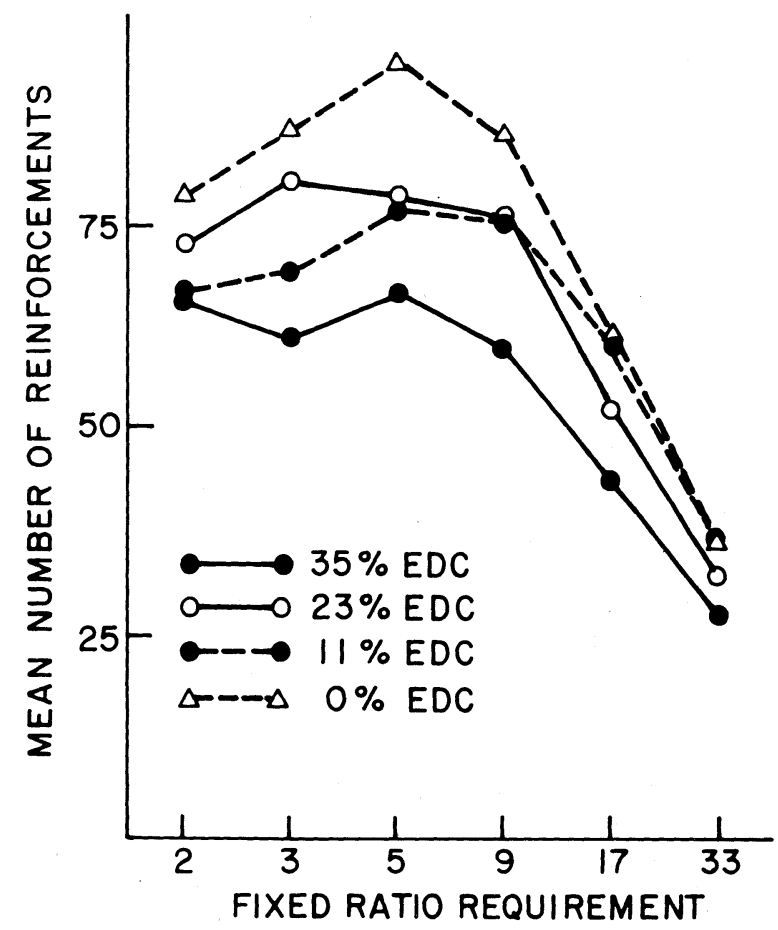

Figure 1. Mean number of reinforcements per 20-min session as a function of FR requirement and maternal alcohol consumption. 


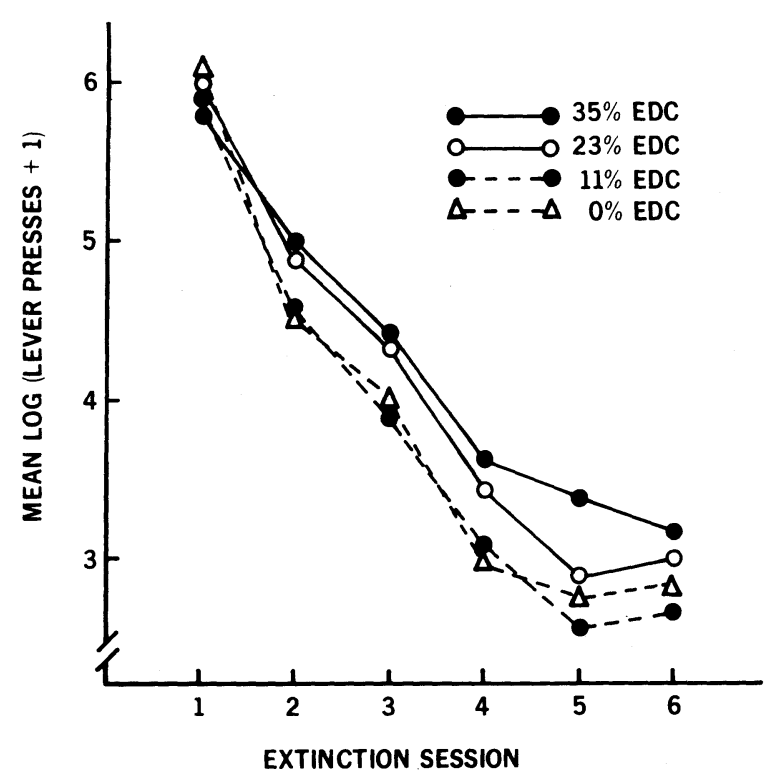

Figure 2. Mean log of number of responses per 20-min session during the extinction phase as a function of maternal alcohol consumption.

across groups in that the interaction of sessions and groups failed to approach significance, $\mathrm{F}<1$.

Separate analyses were conducted to compare the $0 \%$ control group to the lab chow control, since this latter group is not on the same continuum as the liquid-diet groups and its inclusion in the overall analyses would have precluded subsequent trend analyses. In no instance did these two groups differ from each other. During the acquisition phase, there were significant effects of ratio $[F(5,80)=36.32$, $\mathrm{p}<.01]$ and days $[\mathrm{F}(2,32)=26.47, \mathrm{p}<.01]$, but neither of these variables interacted with groups, nor was the main effect of groups significant $(F<1)$. Extinction data were transformed as described previously. During extinction, only the effect of session was found to be significant $[\mathrm{F}(5,80)=167.22, \mathrm{p}<$ $.001]$. The difference between the $0 \%$ and the lab chow control groups did not approach significance, $\mathrm{F}<1$.

Detailed information concerning maternal intakes and the development of all the offspring is being presented elsewhere (Lochry, Shapiro, \& Riley, in press). In brief, daily maternal alcohol consumption averaged $12.82,8.26$, and $4.18 \mathrm{~g} / \mathrm{kg}$ for mothers fed the $35 \%, 23 \%$, and $11 \%$ EDC diets, respectively. Linear dose-response effects were found for offspring body weight and length, where the animals exposed to alcohol evidenced growth deficiencies. Furthermore, there was a higher incidence of perinatal death among the experimental, alcohol-exposed animals. However, analysis of body weights at 20 days of age for the subjects used in the present investigation indicated no significant effect of maternal diet $[F(3,30)$ $=2.63, \mathrm{p}>.05]$. Similarly, the $0 \%$ and lab chow control groups had equivalent body weights at 20 days of age, $\mathrm{F}<1$.

\section{DISCUSSION}

Response rates on several fixed-ratio schedules were found to vary inversely with the extent of alcohol insult in utero. As the schedule requirement was progressively increased from FR 2 to FR 33, the 35\% offspring consistently had the lowest rates, $23 \%$ and $11 \%$ offspring were intermediate, and the $0 \%$ subjects had the highest rates. During extinction, this pattern was reversed; resistance to extinction was directly related to the amount of prenatal alcohol exposure. Furthermore, these behavioral differences between the alcohol-exposed and control offspring occurred in the absence of any obvious physical distinctions.

The acquisition data from this study partially replicate data reported by Martin et al. (1977). They reported that animals exposed to alcohol during both gestation and while nursing received fewer reinforcements on CRF and FR-10 schedules, although differences during the latter part of the FR-10 phase were not significant. The diminished effect reported by Martin et al. (1977), in contrast to the persistent effects reported here, may possibly be due to several factors. In the former study, alcohol was administered both pre- and postnatally and, because a twobar task was used, the schedule was confounded with the appropriately reinforced lever. However, in the present study, the finding of a diminished effect may have been precluded, since the schedule requirement was changed every 3 days.

With regard to our findings, the possibility that lowered response rates in acquisition are the result of motor incoordination in alcohol-exposed progeny seems unlikely, given the fact that these animals were able to increase their response rates when the response requirement was increased. (It should be noted that such increases may not be directly apparent in Figure 1, since increases in response rates generally were not sufficient to maintain the previous rate of reinforcement.) For the most part, rates of alcoholexposed progeny on any of the FR schedules exceeded those of controls on the previous schedule. For example, response rates of alcohol-exposed offspring on the FR-9 schedule represent an increase of $171 \%$ over these same animals' FR-5 rate, and an increase of $133 \%$ over the control $(0 \%)$ group's FR 5 rate.

The lower response rates of alcohol-exposed progeny during acquisition also do not appear to reflect decreased motivational factors on their part. If this was the case, then one would expect to find faster extinction rates among the alcohol-exposed animals (Perin, 1942). Furthermore, these extinction data evade any straightforward interpretation in terms of 
differential preextinction reinforcement experience, since the decreased number of rewards obtained during acquisition in the experimental animals would be expected to produce faster extinction rates (Perin, 1942).

The present data might, however, be explained by proposing that prenatal alcohol exposure affects learning ability such that rats in these groups are slower to pick up on the demands of the FR schedule, and thus have lower acquisition rates for any particular FR schedule. During extinction, prenatally exposed animals would be expected to evidence higher response rates than controls, since they would be slower at detecting the absence of a reinforcement contingency. An alternative explanation of the extinction data involves a suggestion by Riley et al. (1979a, 1979b) that prenatal alcohol exposure causes a deficit in inhibitory tendencies and a propensity to perseverate on prepotent responses. Since extinction requires the active inhibition of responding, and manipulations such as septal lesions, which presumably act as disinhibitors, retard extinction (Schwartzbaum, Kellicutt, Spieth, \& Thompson, 1964), these data are consistent with this interpretation.

This inhibition deficit interpretation also agrees with the behavioral descriptions of children born to alcoholic women. These children have been described as "fidgety, distractible, always on the go, and never sitting still"' (Streissguth, Herman, \& Smith, 1978). However, a response inhibition deficit interpretation obviously cannot account for all the data. For example, animals exposed prenatally to alcohol evidence deficits in shuttle avoidance (Bond \& DiGiusto, 1978; Shaywitz et al., 1976), a finding that is not predicted if these animals are only deficient in response inhibition. More likely, prenatal alcohol exposure results in some type of interference with the functioning of central inhibitory processes as well as causing cognitive dysfunction.

A final note of caution is in order. Because crossfostering procedures were not employed in the present study, the possible role of altered postnatal maternal behavior due to the prenatal alcohol treatment cannot be ruled out. To date, however, those studies involving prenatal alcohol exposure which have utilized crossfostering still find behavioral differences in the treated progeny as opposed to untreated controls (Bond \& DiGiusto, 1978; Martin et al., 1978). It appears, therefore, that alcohol exposure in utero is capable of inducing subsequent behavioral aberrations in the offspring that are independent of any obvious physical abnormalities.

\section{REFERENCES}

Bond, N. W., \& DiGiusto, E. L. Effects of prenatal alcohol consumption and open-field behaviour and alcohol preference in rats. Psychopharmacology, 1976, 46, 163-168.

Bond, N. W., \& DiGiusto, E. L. Prenatal alcohol consumption and open-field behaviour in rats: Effects of age at time of testing. Psychopharmacology, 1977, 52, 311-312.

Bond, N. W., \& DiGiusto, E. L. Avoidance conditioning and Hebb-Williams maze performance in rats treated prenatally with alcohol. Psychopharmacology, 1978, 58, 69-71.

Branchey, L., \& Friedhoff, A. J. Biochemical and behavioral changes in rats exposed to ethanol in utero. Annals of the New York Academy of Sciences, 1976, 273, 328-330.

Demers, M., \& Kirouac, G. Prenatal effects of ethanol on the behavioral development of the rat. Physiological Psychology, $1978,6,517-520$.

Henderson, G. I., \& Schenker, S. The effect of maternal alcohol consumption on the viability and visceral development of the newborn rat. Research Communications in Chemical Pathology and Pharmacology, 1977, 16, 15-32.

KEPPEL, G. Design and analysis: $A$ researchers handbook. Englewood Cliffs, N.J: Prentice-Hall, 1973.

LESTER, D. Rapid methods for processing large numbers of tissue samples of ethyl alcohol. Proceedings of the Third International Conference on Alcohol and Road Traffic. London: British Medical Association, 1963.

Lochry, E. A., Shapiro, N. R., \& Riley, E. P. Growth deficits in rats exposed to alcohol in utero. Journal of Studies on Alcohol, in press.

Martin, J. C., Martin, D. C., Sigman, G., \& Radow, B. Offspring survival, development, and operant performance following maternal ethanol consumption. Developmental Psychobiology, 1977, 10, 435-446.

Martin, J. C., Martin, D. C., Sigman, G., \& Radow, B. Maternal ethanol consumption and hyperactivity in cross-fostered offspring. Physiological Psychology, 1978, 6, 362-365.

Perin, C. T. Behavior potentiality as a joint function of the amount of training and the degree of hunger at the time of extinction. Journal of Experimental Psychology, 1942, 30, 93-113.

Riley, E. P., Lochry, E. A., \& Shapiro, N. R. Lack of response inhibition in rats prenatally exposed to alcohol. Psychopharmacology, 1979, 62, 47-52. (a)

Riley, E. P., Lochry, E. A., Shapiro, N. R., \& Baldwin, J. Response perseveration in rats exposed to alcohol prenatally. Pharmacology, Biochemistry, and Behavior, 1979, 10, 255-259. (b)

Schwartzbaum, J. S., Kellicutt, M. H., Spieth, T. M., \& Thompson, J. B. Effects of septal lesions in rats on response inhibition associated with food-reinforced behavior. Journal of Comparative and Physiological Psychology, 1964, 58, 217-224.

Shaywitz, B. A., Klopper, J. H., \& Gordon, J. W. A syndrome resembling minimal brain dysfunction (MBD) in rat pups born to alcoholic mothers. Pediatric Research, 1976, 10, 451. (Abstract)

Streissguth, A. P., Herman, C. S., \& Smith, D. W. Intelligence, behavior, and dysmorphogenesis in the fetal alcohol syndrome: A report on 20 patients. Journal of Pediatrics, 1978, 92, 363-367.

TzE, W. J., \& LEE, M. Adverse effects of maternal alcohol consumption on pregnancy and foetal growth in rats. Nature, 1975, 257, 479-480.

Wine R, B. J. Statistical principles in experimental design. New York: McGraw-Hill, 1971.

(Received for publication April 10, 1979; revision accepted January $22,1980$. 